\title{
Histopatología en biopsias gástricas y diagnóstico temprano de lesiones premalignas
}

\author{
Felio Palomino, Rogger Verona, Pedro Ruiz, Ernesto Nava, Katherine Vera, Diana Tello
}

Instituto de Patología, Facultad de Medicina, UNMSM

Objetivos: Los diagnósticos histopatológicos oportunos van a permitir el tratamiento clínico y/o quirúrgico en razón del tipo de lesión diagnosticada, buscando mejorar la calidad de vida de los pacientes.

Diseño: Estudio retrospectivo.

Institución: Instituto de Patología, Facultad de Medicina, UNMSM.

Participantes: Pacientes del Hospital Félix Mayorca Soto, Tarma, Huancayo.

Intervenciones: Se ha realizado la revisión macroscópica y microscópica de 300 biopsias gástricas tomadas por endoscopia, en el Hospital Félix Mayorca Soto, de la ciudad de Tarma, Huancayo, cuyo tamaño promedio varió entre 2 y 4 milímetros, los que fueron procesados y coloreados con hematoxilina eosina.

Principales medidas de resultados: Lesiones gástricas benignas y malignas.

Resultados: Del total de biopsias gástricas, $60 \%$ correspondió al sexo femenino y $40 \%$ al masculino. La gastritis crónica moderada ha sido la lesión más frecuente, en 206 (69\%) pacientes. La presencia de Helicobacter pylori fue diagnosticada en $144(48 \%)$ pacientes. De los 300 casos revisados, en $6(2 \%)$ se diagnóstico carcinoma gástrico. El diagnóstico de metaplasia intestinal en diferentes estadios se realizó en $107(24 \%)$ pacientes.

Conclusiones: De las 300 biopsias, $60 \%$ correspondió al sexo femenino y $40 \%$ al masculino. La gastritis crónica moderada ha sido la lesión más frecuente, en $69 \%$ de pacientes, y el Helicobacter pylori fue diagnosticado en $48 \%$ pacientes. Se encontró metaplasia intestinal en diferentes estadios en 38\% y se diagnosticó $6(2 \%)$ casos de carcinoma gástrico.

Palabras clave: Biopsias, gastritis, Helicobacter pylori, metaplasia.

\section{Comparación del efecto hipolipemiante del aceite de sacha inchi con la simvastatina, en diabéticos tipo 2}

Fausto Garmendia, Gerardo Ronceros, Pedro Muro, Rosa Pando Instituto de Investigaciones Clínicas, Facultad de Medicina, UNMSM

Objetivos: Evaluar el efecto hipolipemiante del aceite de sacha inchi.

Diseño: Estudio experimental, doble ciego, con grupo control comparativo.

Institución: Instituto de Investigaciones Clínicas, Facultad de Medicina, UNMSM.

Participantes: Pacientes diabéticos tipo 2 con dislipoproteinemia.

Intervenciones: Se ingresó al estudio a 48 pacientes diabéticos tipo 2 con dislipoproteinemia, de ambos géneros, de 40 a 70 años de edad, sin obesidad mórbida. Mediante una lista aleatoria, 15 recibieron simvastatina $20 \mathrm{mg}+$ aceite placebo (grupo 1) y 14 aceite de sacha inchi +1 comprimido placebo (grupo 2), durante 4 meses. En ayunas, se midió colesterol total (CT), colesterol HDL (HDL), triglicéridos (Tg), glucosa (G) y hemoglobina glicosilada $(\mathrm{HbA} 1 \mathrm{c})$, por métodos enzimáticos convencionales.

Principales medidas de resultados: VLDL y LDL y colesterol no-HDL.

Resultados: En el análisis de muestras pareadas, se apreció una significativa disminución de las concentraciones de CT, LDL, VLDL, no-HDL, Tg y AGNE, con la dosis de $5 \mathrm{~mL}$ de aceite, y una elevación muy importante del colesterol HDL. Con la dosis de $10 \mathrm{~mL}$ hubo disminución significativa del CT, LDL, no-HDL y AGNE, e incremento de HDL, a los cuatro meses de tratamiento; no se encontró disminución de significativa de Tg; hubo elevación leve de glucosa, insulina y el indice HOMA.

Conclusiones: Los resultados preliminares muestran una disminución de CT, LDL y colesterol No-HDL e incremento de $\mathrm{HDL}$ en ambos grupos. Las concentraciones de glucosa y Hba1c no mostraron modificaciones significativas.

Palabras clave: Aceite de sacha inchi, sinvastatina. 